\title{
Uso de Guía de Metil-metacrilato Fabricados en Biomodelo de Poliuretano para el Tratamiento Tardío de Fractura Panfacial
}

\author{
The Use of Metil-metacrilate Guide Manufactured on Biomodel \\ of Polyurethane for Late Treatment of Panfacial Fracture
}

\author{
Leandro Pozzer*; Lucas Cavalieri-Pereira*; Sergio Olate" ${ }^{\star *}$ Luciana Asprino* \& Marcio de Moraes*
}

POZZER, L.; CAVALIERI-PEREIRA, L.; OLATE, S.; ASPRINO, L. \& DE MORAES, M. Uso de guía de metil-metacrylato fabricados en biomodelo de poliuretano para el tratamiento tardío de fractura panfacial. Int. J. Odontostomat., 7(1):73-78, 2013.

RESUMEN: El manejo de las secuelas faciales por fracturas no tratadas ha tenido un crecimiento importante debido al uso de los biomodelos. Estos permiten optimizar la cirugía, disminuir los tiempos quirúrgicos y mejorar los resultados. El presente articulo muestra un caso de secuela de fractura facial tratada con osteotomías con ayuda de guías quirúrgicos fabricados en base a un biomodelo operado; se presenta el resultado postoperatorio de 6 meses con una resolución adecuada.

PALABRAS CLAVE: fractura panfacial, secuela facial, biomodelo.

\section{INTRODUCCIÓN}

El tratamiento de las fracturas panfaciales es una patología altamente compleja y aun mas cuando es realizado de forma tardía (He et al., 2007). Pacientes con secuelas de trauma facial normalmente presentan alteración de la oclusión, ensanchamiento facial, enoftalmo traumático, distopia, entre otros; estas secuelas de trauma presentan generalmente mal unión ósea y contracción cicatricial de diferentes tejidos.

Basados en técnicas para preservar la función y la estética facial, existen muchas formas de realizar el tratamiento quirúrgico, direccionando el procedimiento desde inferior a superior y viceversa, siendo además centrifuga o centrípeta (Kelly, 1978; Gruss \& Phillips, 1989). Algunos autores han sugerido que la reconstrucción facial debería comenzar por la mandíbula para devolver ancho y proyección sagital del tercio inferior (Wenig, 1991) y cuando existe fractura transversal de maxila, se debería comenzar por esta fractura para guiar la reconstrucción mandibular (Kelly et al., 1990). Otros autores han señalado, sin embargo, que el tratamiento debería comenzar por la orientación del arco zigomático y la reconstrucción orbitaria a fin de establecer ancho y proyección facial (Gruss \& Phillips; Gruss et al., 1990); también se ha señalado el comienzo del tratamiento por la fractura naso-orbitoetmoidal (NOE) a fin de restablecer el sector mediano y posteriormente el lateral (Meville, 1974).

Aunque no existe consenso en cual seria la mejor orientación del tratamiento, cualquiera de ellas debe ser acompañada por tomografías computadorizadas y, actualmente, por el apoyo de biomodelos.

El objetivo de este articulo es evidenciar el empleo del biomodelo facial y el apoyo de guías quirúrgicos individualizados a base de resina acrílica empleados en la reconstrucción facial de una paciente con secuela de una fractura panfacial.

* División de Cirugía Oral y Maxilofacial, Facultad de Odontología de Piracicaba, Universidad Estadual de Campinas, Piracicaba, Brasil. ** Unidad de Cirugía Oral y Maxilofacial, Departamento de Odontología Integral Adultos, Facultad de Odontología, Universidad de La Frontera, Chile. 


\section{CASO CLINICO}

Paciente, sexo femenino, 42 años, victima de accidente automovilístico se presento en la admisión con un politrauma facial severo. La paciente presento complicaciones sistémicas asociadas a trombosis venosa profunda y un extenso periodo de asistencia en la unidad de cuidados intensivos y la unidad de tratamiento intensivo.

El diagnostico inicial presentaba fractura de mandíbula, fractura de maxila, fractura de complejo zigomático bilateral con perdida de sustancia en el piso orbitario, fractura NOE y fractura de arco zigomático bilateral. Además, la paciente evoluciono con distopia del ojo derecho y amaurosis del mismo.

Después de 12 meses de evolución del trauma facial (Fig. 1), inicialmente con tratamiento parcial mediante técnicas no quirúrgicas, la paciente presentaba quejas respecto a la conformación de la nariz, posicionamiento del bulbo ocular y el ensanchamiento facial. Al examen presentaba oclusión dentaria satisfactoria y sin alteración funcional, perdida de proyección sagital de la cara, telecanto traumático, distopia, enoftalmo, irregularidades en el dorso nasal y amaurosis del ojo derecho

Se solicito el examen con tomografía computadorizada (Fig. 2) y la confección de un bio- modelo (CTI - Centro de Tecnologia e Informação-Instituto Renato Archer - Campinas, São Paulo, Brasil).

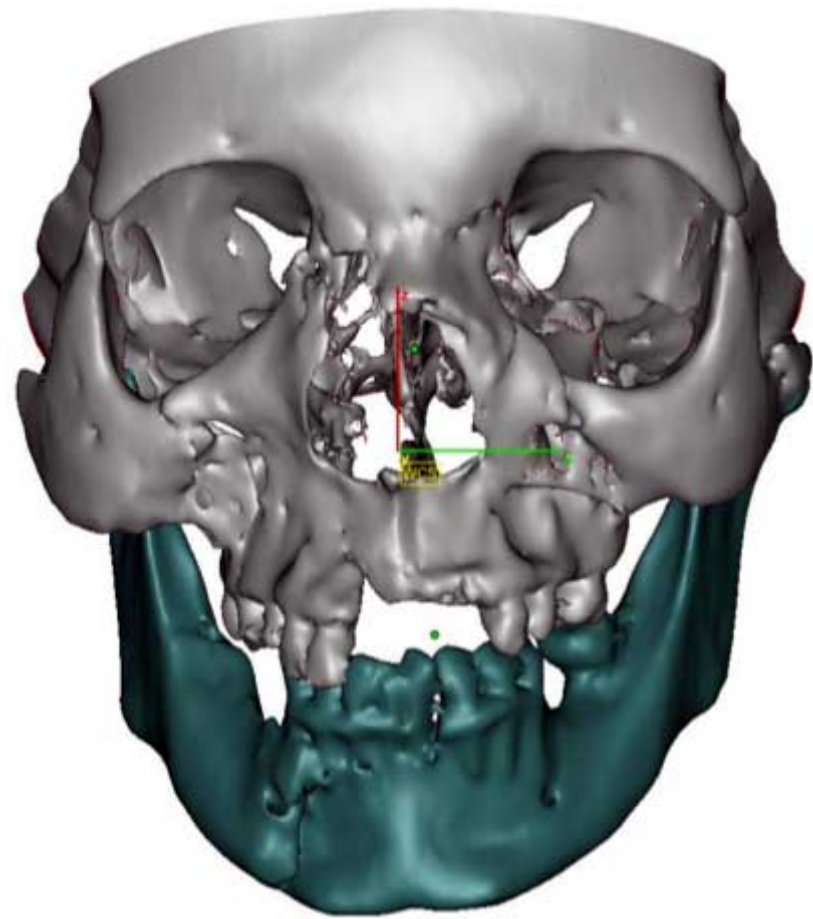

Fig. 2. Reconstrucción volumétrica presentando la deformidad causada por las fracturas múltiples faciales consolidadas en lugares inadecuados.

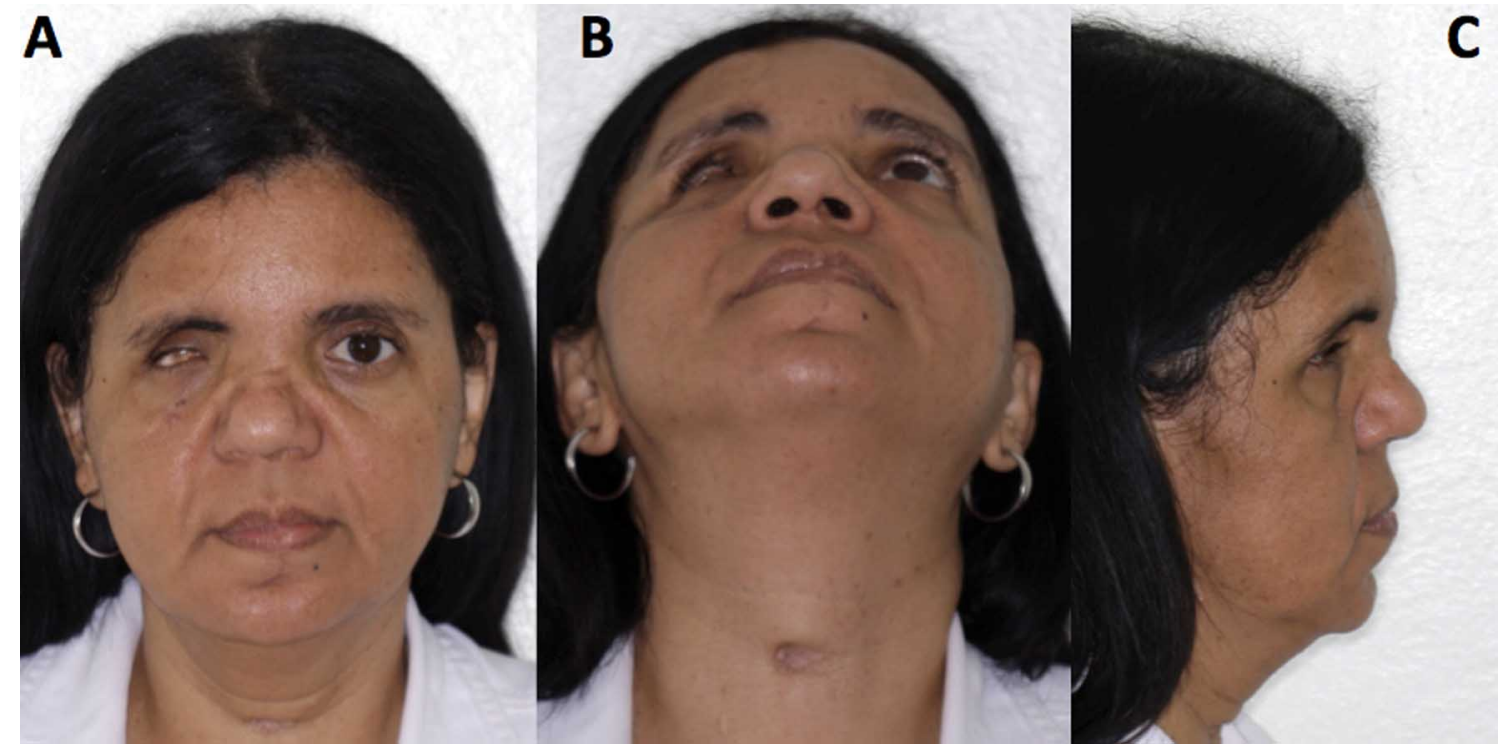

Fig. 1. Condición facial 12 meses después del trauma inicial. A. Vista frontal con perdida de sustancia en sector facial derecho y notable asimetría facial; existe ensanchamiento de la base nasal y telecanto traumático. B. Visión submentoniana demostrando la perdida de proyección anterior del área paranasal y cigomática izquierda. C. Visión lateral mostrando defecto en silla, característicos de las fracturas naso-orbito-etmoidales 


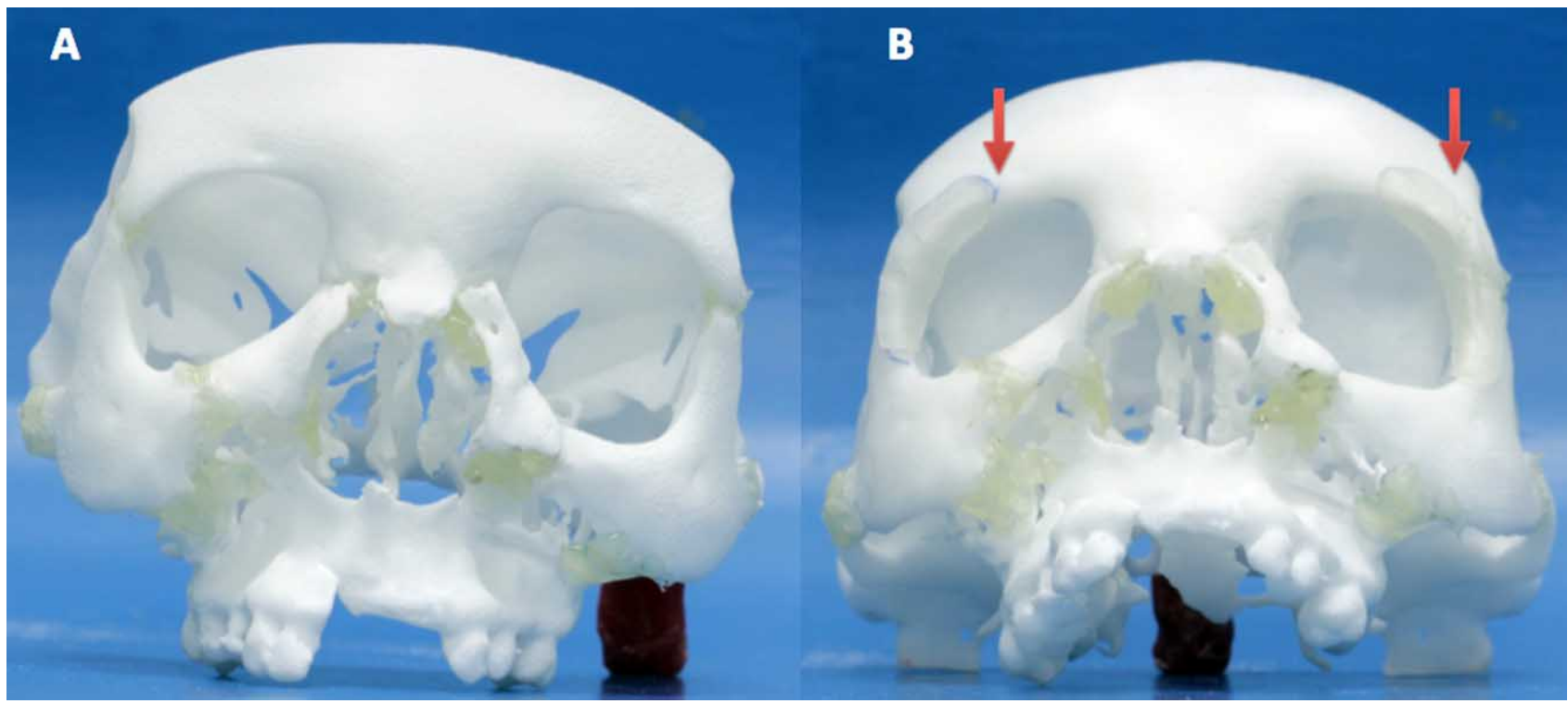

Fig. 3. Biomodelos del tercio medio facial. A. Osteotomías realizadas en los deferentes sectores orbitarios, huesos propios de nariz, complejo zigomático orbitario: la reposición se realizo con sistemas adhesivos buscando simetría facial y orbitaria. B. Confección de guías quirúrgicos de resina acrílica posicionados en el sector superior y lateral de la órbita.

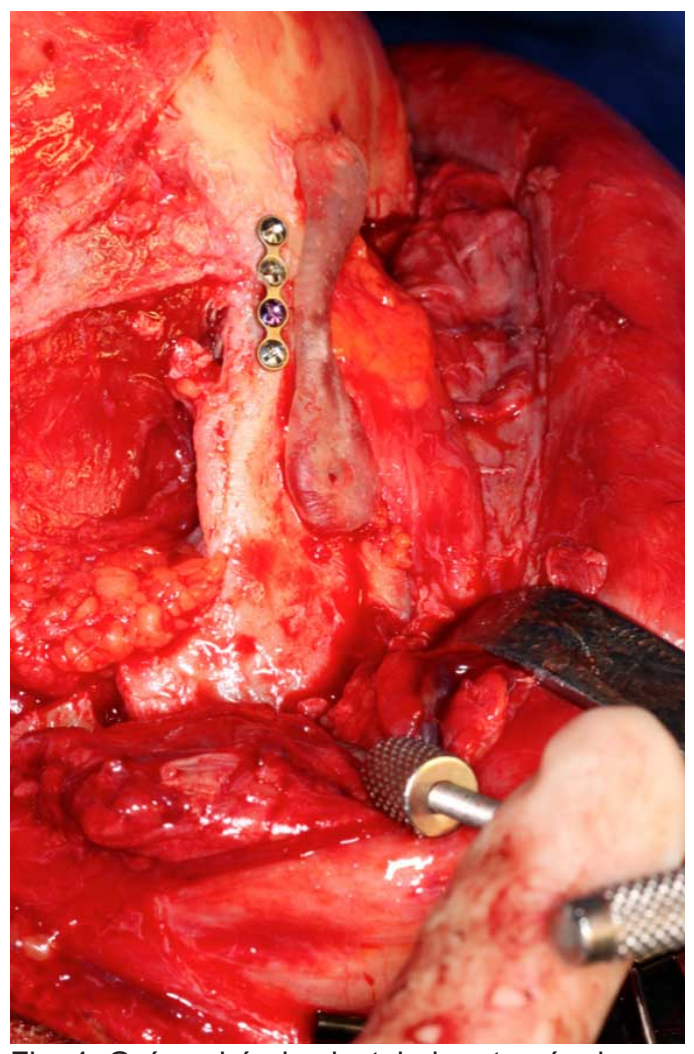

Fig. 4. Guía quirúrgico instalado a través de una acceso coronal. El guía es fijados con tornillos durante la etapa intraoperatoria, antes de la instalación de la osteosíntesis de la sutura frontocigomatica. Se observa el tornillo de CarollGirard para la movilización del cuerpo de hueso cigomático.
La tomografía evidencio que la consolidación ósea producida en maxila y mandíbula fue satisfactorio, toda vez que el manejo no quirúrgico inicial fue orientado al manejo estable de la oclusión. De esta forma, la indicación quirúrgica se planteo para el manejo de las lesiones del tercio medio facial.

El biomodelo fue utilizado para planificar la reposición ósea adecuada (Fig. 3), realizando la segmentación ósea en las suturas con mala posición. Esta reposición busco simetría facial y la reconstrucción de las paredes orbitarias de forma simétrica. También se estimo la segmentación de los huesos propios de la nariz y la reposición del telecanto traumático mediante cantopexia. Al segmentar los fragmentos en el biomodelo, se realizaron guías quirúrgicos en base a resina acrílica que permitirían posicionar los fragmentos de forma adecuada.

Sobre anestesia general, con intubación orotraqueal, se realizo la intervención a través de un acceso coronal, subciliar bilateral e intraoral bilateral. Todas las estructuras consolidadas fueron osteotomizadas con fresas y sierra reciprocante. Se intervino bilateralmente los arcos zigomáticos, sutura frontocigomática, reborde infraorbitario y pilar cigomático además de la sutura nasofrontal y de los huesos propios de la nariz.

Luego de las osteotomías, un tornillo de Carrol-Girard fue posicionado en el cuerpo del hueso cigomático para una manipulación correcta. Se comenzó a intervenir en el sector lateral derecho posicionando el guía de reducción orbitario (Fig. 4) y la posterior instalación de una placa del sistema 2.0 en la sutura frontocigomática y a continuación en el arco zigomático 
POZZER, L.; CAVALIERI-PEREIRA, L.; OLATE, S.; ASPRINO, L. \& DE MORAES, M. Uso de guía de metil-metacrylato fabricados en biomodelo de poliuretano para el tratamiento tardío de fractura panfacial. Int. J. Odontostomat., 7(1):73-78, 2013.

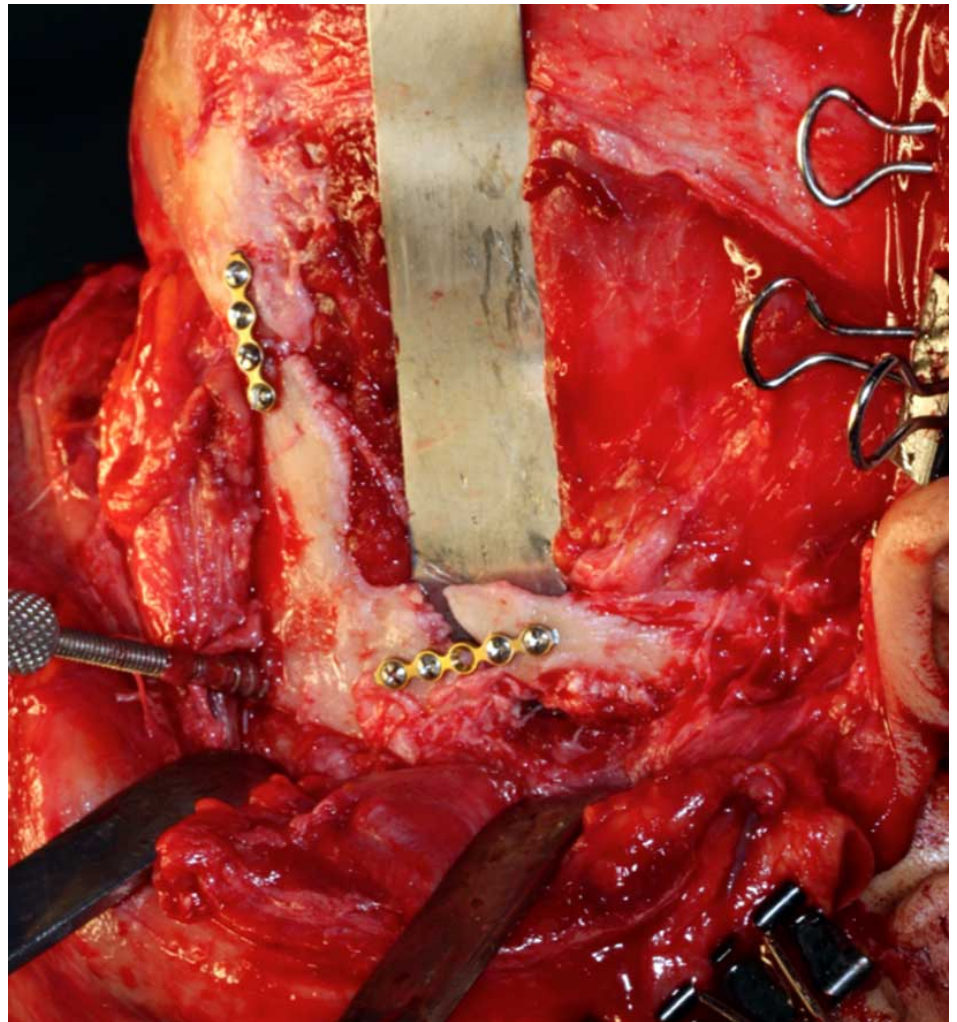

Fig. 5. El guía quirúrgico también permite la instalación de la placa en el arco zigomático, reconstruyendo la proyección sagital facial.

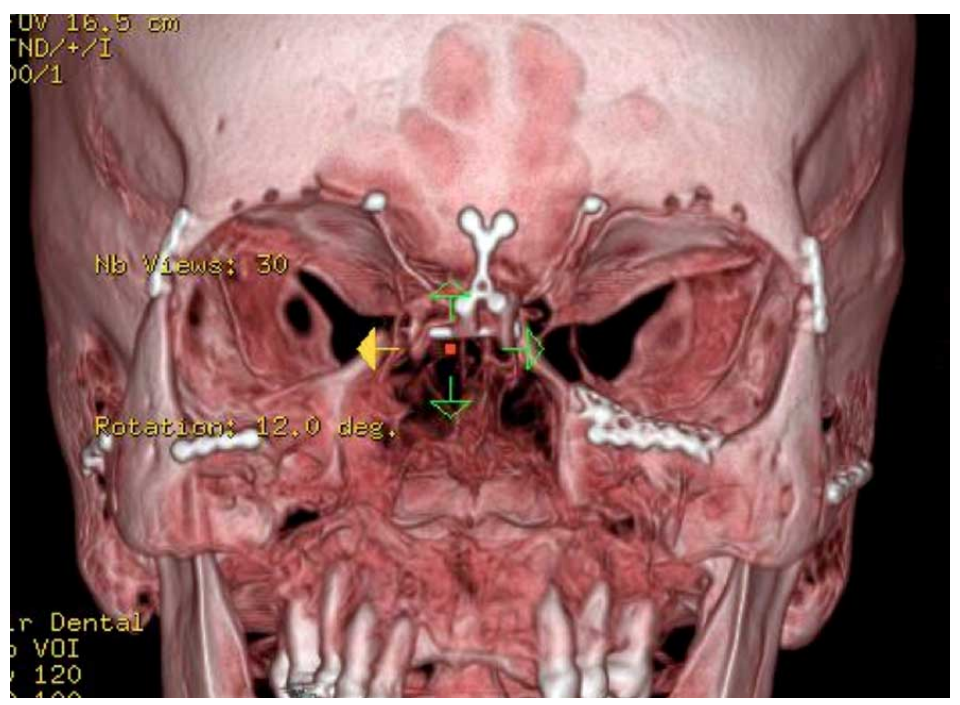

Fig. 6. Imagen tomografía postoperatoria que muestra los sistemas de osteosíntesis instalados con una adecuada reposición ósea y un volumen orbitario simétrico

derecho (Fig. 5); este guía también permitió la fijación de otros sectores orbitarios. La reconstrucción orbitaria se realizo con mallas de titanio. Esta misma secuencia se utilizo para la reducción y osteosíntesis del lado izquierdo con su respectivo guía quirúrgico.
La secuencia continuó con la reducción de la estructura nasal mediante la instalación de una placa en " $x$ " del sistema 2.0 que permitía la correcta reposición de la osteotomía NOE; el contorno nasal final se obtuvo mediante el uso de un implante facial aloplástico (polietileno poroso) ubicado en el dorso nasal (Figura 6). Finalmente se realizo la cantopexia bilateral, la suspensión de la musculatura supraorbitaria mediante técnicas de sutura y el cierre de los accesos quirúrgicos.

La imagen tomográfica posterior demostró una adecuada restauración del ancho facial y de la proyección anteroposterior facial (Fig. 7), sin alteraciones en la posición ósea; se consiguió una adecuada restauración de los tejidos duros. El seguimiento de la paciente, después de seis meses, ha sido favorable, con una adecuada restauración facial y reposición ósea de los fragmentos faciales mal orientados; además, como parte de su rehabilitación facial, fue incorporada una prótesis ocular del ojo izquierdo que permite mimetizar perfectamente la lesión generada en este sector (Fig. 8).

\section{DISCUSIÓN}

No existe consenso en el tratamiento de las fracturas panfaciales. De hecho, hasta la misma definición de fractura panfacial puede ser dudoso toda vez que se ha sugerido que este tipo de lesiones debería necesariamente involucrar el tercio medio e inferior, mientras que otros sostienen la necesidad de incluir también el tercio superior (Wenig).

La gran deficiencia al tratar fracturas faciales tardías esta en la contracción cicatricial y la perdida de elasticidad de los tejidos; de hecho, después de tres semanas de ocurrida la fractura, ya es posible observar estados iniciales de consolidación y avanzada remodelación ósea de forma que la reducción quirúrgica se hace altamente compleja; esto implica, generalmente, mal unión, maloclusión, defectos óseos y en algunos casos pseudoartrosis (Carr \& Methog, 1997).

La planificación quirúrgica de esta frac- 


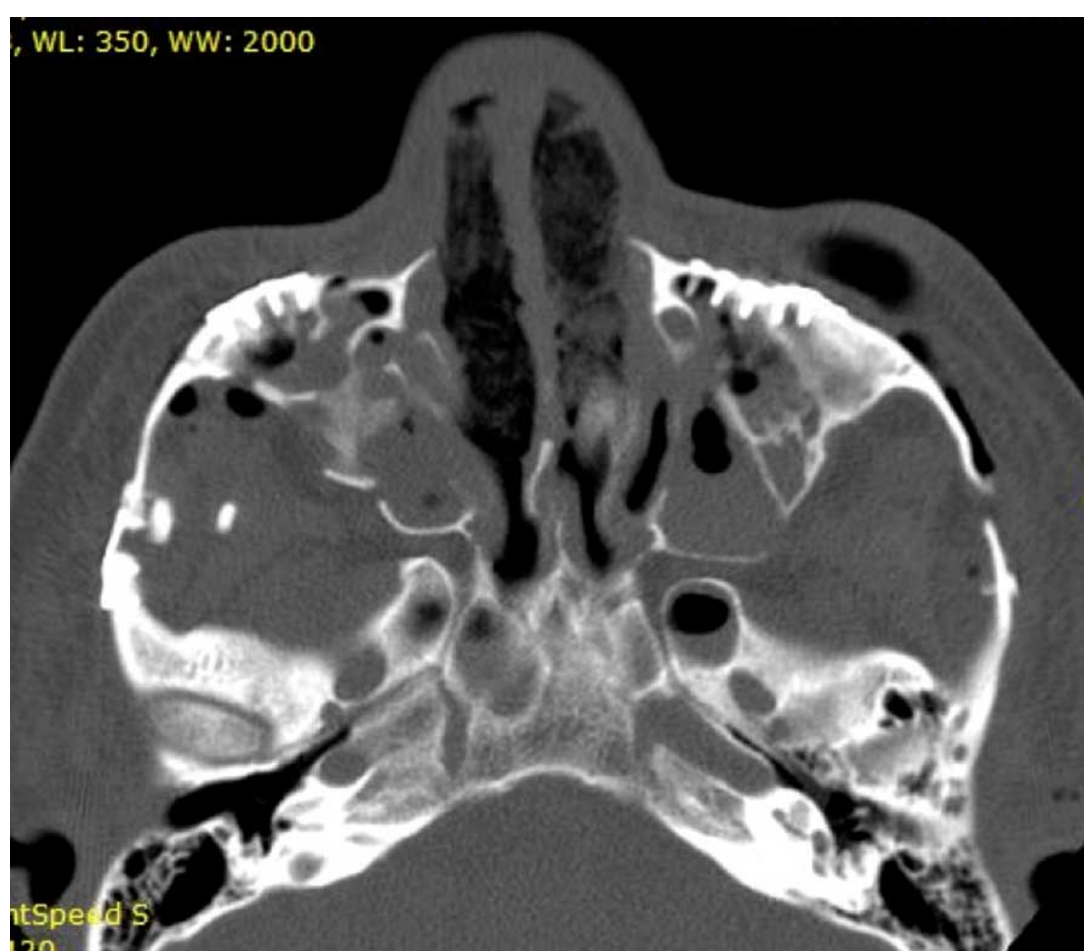

Fig. 7. Corte coronal de la tomografía computadorizada donde se observa una adecuada restauración anteroposterior del arco zigomático junto a un ancho facial adecuado y simétrico.

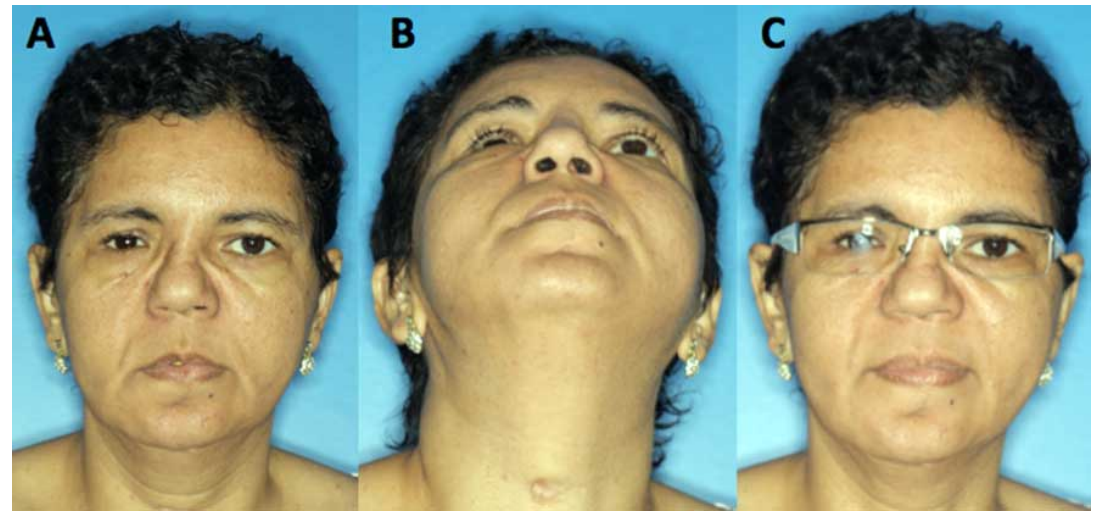

Fig. 8. Postoperatorio después de 6 meses. A. Visión frontal observando simetría facial y adecuada restauración de la distancia intercantal; existen deficiencias en la punta nasal que se podrán solucionar futuramente con procedimientos menores. B. Visión submentoniana demostrando una adecuada proyección anteroposterior y buena posición de la prótesis ocular derecha. C. Visión frontal con lentes, utilizados generalmente para mimetizar el efecto de la prótesis del bulbo ocular.

turas es altamente compleja; los biomodelos han optimizado esta planificación, ya que permiten ensayar diferentes tipos de cirugías para conseguir los objetivos trazados y la reciente incorporación de guías quirúrgicos de resina acrílica, de fácil elaboración, permiten optimizar la cirugía, escoger el sistema de osteosíntesis y realizar su adaptación antes de la cirugía, disminuyendo el tiempo quirúrgico y mejorando los resultados ( $\mathrm{He}$ et al.).
El uso de imagen 3D y biomodelos, sin duda, optimiza la cirugía reconstructiva y permiten que estos guías sean utilizados a fin de un mejor resultado; permiten definir incluso la posición mas adecuada de la osteotomía (Mayeink et al., 2011).

Finalmente, podemos concluir que la secuela de fracturas faciales complejas pueden ser abordados y optimizados con el empleo de guías quirúrgicos basados en la reconstrucción con biomodelos, permitiendo resultados bastantes próximos de los inicialmente planificados.

POZZER, L.; CAVALIERI-PEREIRA, L.; OLATE, S.; ASPRINO, L. \& DE MORAES, M. The use of metil-metacrilate guide manufactured on biomodel of polyurethane for late treatment of panfacial fracture. Int. J. Odontostomat., 7(1):73-78, 2013.

ABSTRACT: The management of facial sequel by facial fractures nontreated nowadays presents an important expansion by the use of biomodels. This system can be used for reduce surgical time, optimize surgical protocol and improve outcomes. This paper presents a sequel case for facial fracture treated with bone osteotomy using surgical guide manufactured base on biomodels operated; is showing the postoperative 6 month follow-up with good results.

KEY WORDS: panfacial fracture, facial sequel, biomodel.

\section{REFERENCIAS BIBLIOGRÁFICAS}

Carr, R. M. \& Mathog, R. H. Early and delayed repair of orbitozygomatic complex fractures. J. Oral Maxillofac. Surg., 55(3):253-8, 1997.

Gruss, J. S. \& Phillips, J. H. Complex facial trauma: The evolving role of rigid fixation and immediate bone graft reconstruction. Clin. Plast. Surg., 16(1):93-104, 1989. 
POZZER, L.; CAVALIERI-PEREIRA, L.; OLATE, S.; ASPRINO, L. \& DE MORAES, M. Uso de guía de metil-metacrylato fabricados en biomodelo de poliuretano para el tratamiento tardío de fractura panfacial. Int. J. Odontostomat., 7(1):73-78, 2013.

Gruss, J. S.; Van Wyck, L. V.; Phillips, J. H. \& Antonyshyn, $\mathrm{O}$. The importance of the zygomatic arch in complex midfacial fracture repair and correction of posttraumatic orbitozygomatic deformities. Plast. Reconstr. Surg., 85(6):878-90, 1990.

He, D.; Zhang, Y. \& Ellis, E. 3rd. Panfacial Fractures: Analysis of 33 Cases Treated Late. J. Oral Maxillofac. Surg., 65(12):2459-65, 2007.

Kelly, J. War injuries to the jaws and related structures. Washington DC, US Government Printing Office, 1978.

Kelly, K. J.; Manson, P. N.; Vander Kolk, C. A.; Markowitz, B. L.; Dunham, C. M.; Rumley, T. O. \& Crawley, W. A. Sequencing LeFort fracture treatment (Organization of treatment for a panfacial fracture). J. Craniofac. Surg., 1(4):168-78, 1990

Mayrink, G.; Asprino, L.; Moreira, R. W. F.; Paschoal, G. H. L.; Noritomi, P. \& de Moraes, M. Using biomodels for maxillofacial surgeries: 10 years of experience in a Brazilian public service. Braz. J. Oral Sci., 10(4):294-6, 2011.

Merville, L. Multiple dislocations of the facial skeleton. J. Maxillofac. Surg., 2(4):187-200, 1974.

Wenig, B. L. Management of panfacial fractures. Otolaryngol. Clin. North Am., 24(1):93-101, 1991.
Dirección para correspondencia

Prof. Dr. Márcio de Moraes

Faculdade de Odontologia de Piracicaba - FOP/Unicamp

Avenida Limeira, 901

Bairro: Areião

CEP: $13.414-903$

Piracicaba - São Paulo

BRASIL

Email: leandropozzer@yahoo.com.br sergio.olate@ufrontera.cl

Recibido : 26-05-2012

Aceptado : 19-12-2012 